\title{
Melanoma maligno primario atípico de esófago y su manejo quirúrgico: a propósito de un caso clínico
}

\author{
Aníbal Ibáñez ${ }^{1}$, Camila Seymour ${ }^{1}$, Francisco Izquierdo ${ }^{1}$ y Juan Stambuk ${ }^{1}$
}

'Equipo de Cirugía Oncológica, Hospital San Juan de Dios, Santiago, Chile.

Recibido el 24 de noviembre de 2016, aceptado el 10 de enero de 2017

Correspondencia a: Camila Seymour cseymourm@gmail.com

\section{Atypical primary malignant melanoma of esophagus and the surgical management: Case report}

Introduction: The Primary Malignant Melanoma of Esophagus is an uncommon and very agressive tumor. With controversial management, it predominates the surgical treatment in localized disease. Clinical case: We present the case of a young women with Primary Malignant Melanoma of Esophagus with atypical presentation and its surgical management using the Akiyama technique.

Key words: Melanoma of esophagus; Akiyama; Esophagectomy.

\section{Resumen}

Introducción: El melanoma maligno primario de esófago es un tumor raro y muy agresivo. De manejo controversial, el tratamiento quirúrgico es el que predomina en enfermedad localizada. Caso clínico: Presentamos el caso de una mujer joven con melanoma maligno primario de esófago de presentación atípica y su manejo quirúrgico utilizando la técnica de Akiyama.

Palabras clave: Melanoma de esófago; Akiyama; Esofagectomía.

\section{Introducción}

El melanoma maligno primario de esófago (MMPE) es un tumor muy raro y agresivo, que representa el $0,1-0,2 \%$ de los cánceres esofágicos, y el $0,5 \%$ de los melanomas viscerales ${ }^{1,2}$. Su pronóstico es ominoso, con una sobrevida de un $30 \%$ a un año, y de un $5 \%$ a 5 años ${ }^{2}$.

En la literatura existen pocos casos reportados de esta patología, encontrándose mayoritariamente series de algunos casos y análisis retrospectivos. Es por esto que el manejo óptimo del MMPE es incierto, sin embargo, la esofagectomía ha mostrado sobrevidas razonables en enfermedad localizada ${ }^{3}$.

A continuación presentamos el caso clínico de una mujer con MMPE de presentación atípica y que es manejada de forma quirúrgica, junto con una revisión de la literatura existente.

\section{Caso clínico}

Paciente de sexo femenino de 52 años, sin antecedentes mórbidos; presenta un cuadro caracteri- zado por disfagia lógica progresiva de 3 meses de evolución asociada a baja de peso, y examen físico sin hallazgos patológicos. Se solicitó endoscopia digestiva alta que informó 2 lesiones de aspecto similar, blanquecinas, grandes, exofíticas y friables, localizadas a 25 y $30 \mathrm{~cm}$ de la arcada dentaria (Figura 1). Biopsia compatible con melanoma maligno; inmunohistoquímica positiva para S-100 y HMB-45, y negativa para citoqueratina y CEA. Se solicitó PET-CT que informó engrosamiento esofágico medio y distal de $9 \mathrm{~cm}$ de largo, sin evidencias de linfonodos hipercaptantes ni localización secundaria (Figura 2). Fibrobroncoscopia sin lesiones.

Se presenta el caso en comité oncológico. Dadas las buenas condiciones generales de la paciente, su edad y enfermedad localizada, se decide el manejo quirúrgico con criterio oncológico.

La paciente ingresó para cirugía programada. Se realizó esofagectomía transtorácica con linfadenectomía de 3 campos y reconstitución del tránsito con una plastía gástrica ascendida por vía mediastínica (técnica de Akiyama). La paciente evolucionó con fístula esofagogástrica cervical, de manejo 


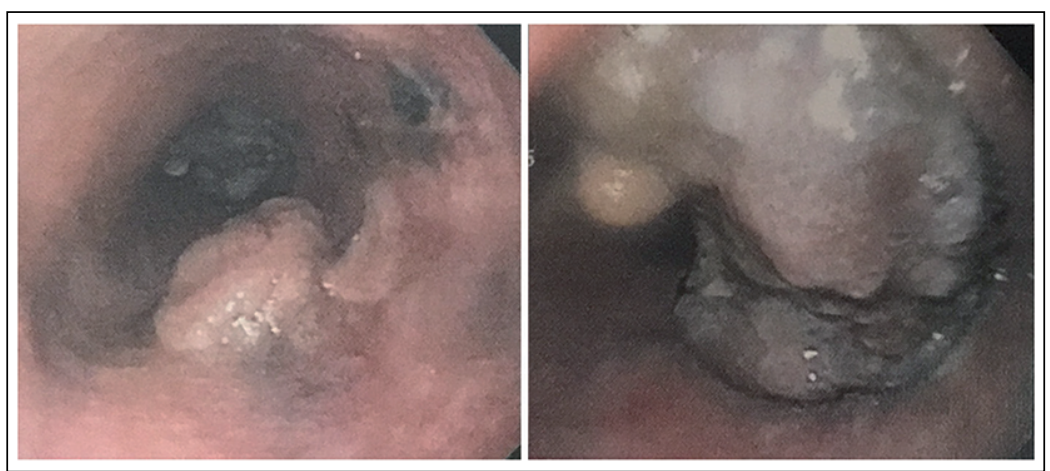

Figura 1. Endoscopia digestiva alta que muestra 2 lesiones exofíticas y friables a 25 y $30 \mathrm{~cm}$ de la arcada dentaria.

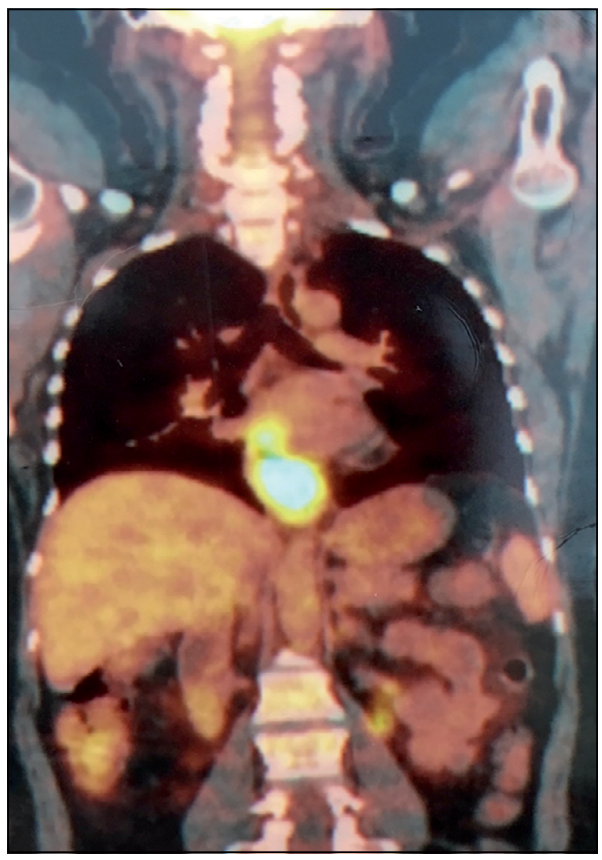

Figura 2. PET-CT que muestra engrosamiento hipercaptante de esófago medio y distal.

conservador y cierre espontáneo. La biopsia diferida informa tumor bilobulado blanquecino grisáceo de $4,5 \times 4 \times 3 \mathrm{~cm}$, a $3 \mathrm{~cm}$ del borde proximal y a $3,5 \mathrm{~cm}$ del borde distal, localizado en la pared posterior. Histología de neoplasia maligna esofágica poco diferenciada, constituida por células de núcleos grandes con nucléolo prominente y pigmento citoplasmático. Inmunohistoquímica positiva para S-100 y HMB-45, negativa para CD-45 y pancitoqueratina (Figura 3), y compatible con MMPE. El estudio ganglionar resultó negativo para neoplasia.

Se presentó nuevamente en comité oncológico, decidiéndose solo la observación seriada. Actualmente la paciente lleva un seguimiento de 12 meses en buenas condiciones y sin evidencias de recidiva.
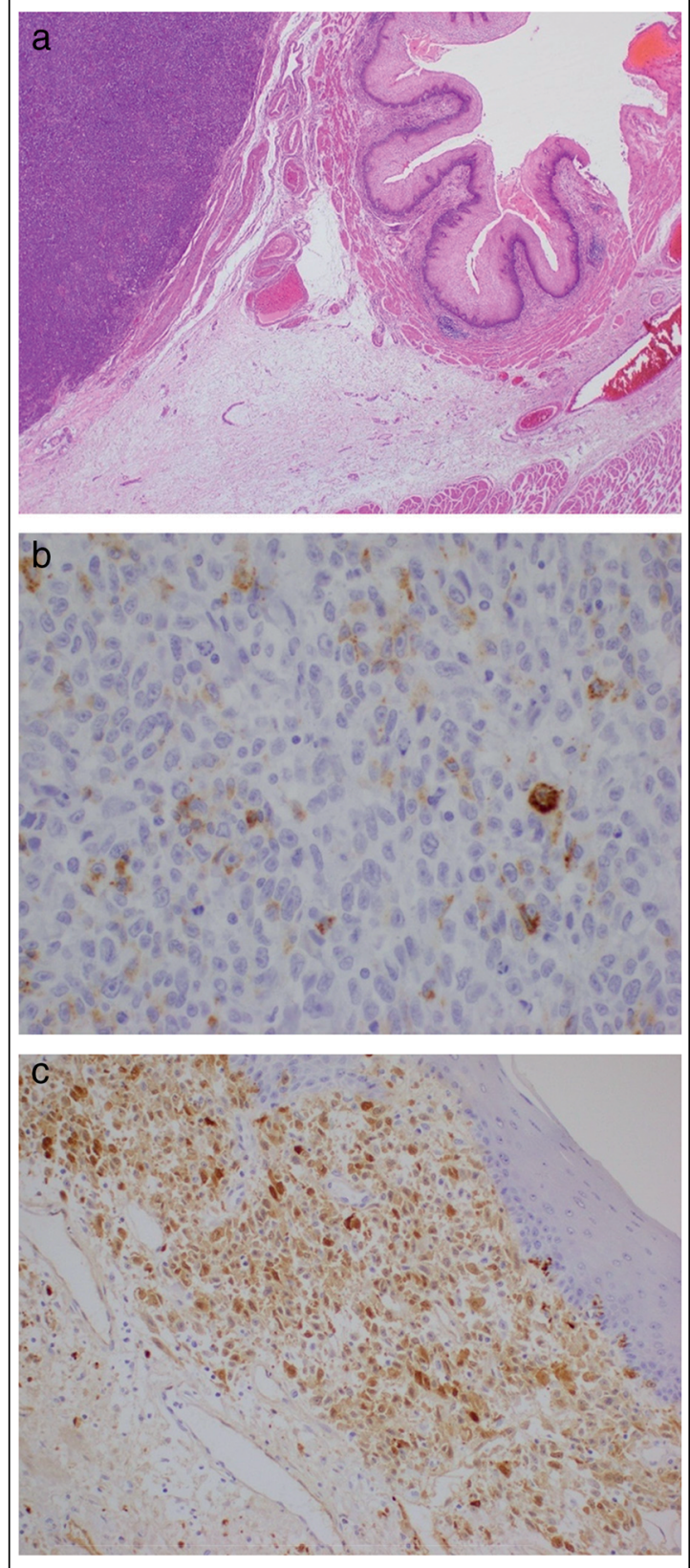

Figura 3. Estudio histológico de la pieza quirúrgica: a) hematoxilina-eosina; b) HMB-45; c) S-100.

\section{Discusión}

El MMPE es un tumor muy raro y agresivo, encontrándose apenas una sobrevida de un $30 \%$ a un año. Los casos reportados son limitados. En nuestro país, solo existen 2 reportes publicados de 3 casos clínicos, en los cuales se realizó esofagectomía ${ }^{4,5}$. 
Su fisiopatología es incierta, sin embargo, se ha propuesto la melanocitosis como un factor de riesgo $^{6}$. Se presenta en la sexta década de la vida, con una relación hombre:mujer de 2:17.

En general, tiene una corta duración de sus síntomas, con una clínica muy similar a otro tipo de tumores de esta localización, manifestándose principalmente con disfagia y dolor retroesternal inespecífico; menos frecuente es la hematemesis o melena $^{1,2,8,9}$.

$\mathrm{Su}$ estudio se inicia con endoscopia digestiva alta, identificándose como una lesión polipoidea, intraluminal, y generalmente pigmentada ${ }^{10,11}$. En un $90 \%$ de los casos, se localiza en el tercio medio o distal del esófago, usualmente como un tumor único. En nuestro caso se encontraron 2 lesiones. En la literatura se encuentran reportes de múltiples lesiones descritas en un $12 \%$ de los $\operatorname{casos}^{2,12}$. Sin embargo, su color no era característico, dificultando su diagnóstico.

El diagnóstico definitivo se realiza a través del estudio histopatológico, incluyendo el análisis inmunohistoquímico. En nuestro caso, el estudio identificó la presencia de gránulos de melanina en el tumor, y la inmunohistoquímica fue compatible con melanoma, siendo positivo para S-100 y HMB-45.

Como otros melanomas, el MMPE es muy agresivo, con una gran tendencia a invadir en profundidad, y con diseminación por vías sanguínea y linfática. Así, se describe diseminación hacia las cadenas ganglionares locorregionales (torácica, tronco celíaco y supraclaviculares izquierda), mediastínicos $\mathrm{y}$, en orden decreciente, hacia hígado, pulmón, pleura, peritoneo, cerebro y hueso ${ }^{9,12,13}$. Al momento del diagnóstico se encuentra un $40 \%$ con metástasis. Para estadificar el MMPE se han usado distintas técnicas. En este caso el estudio con PETTC y fibrobroncoscopia, además de la ausencia de lesiones pigmentarias en el resto del cuerpo, mostró la ausencia de enfermedad diseminada.

No existe consenso sobre el tratamiento del MMPE debido a su rareza. La tendencia actual es el tratamiento quirúrgico cuando no presenta enfermedad metastásica ni compromiso linfonodal extenso. La esofagectomía con linfadenectomía de 3 campos es la técnica que utilizamos en el tratamiento de los carcinomas espinocelulares esofágicos, y es la que aplicamos en este caso, convencidos del valor de la disección linfática extensa ${ }^{2}$. En el tratamiento quirúrgico de los melanomas en general, la técnica del linfonodo centinela ha reemplazado en los casos catalogados como etapa I a la linfadenectomía profiláctica. En los casos con linfonodo centinela negati- vo (estudiados con tinciones de hematoxilina-eosina e inmunohistoquímica), y empleando en su localización intraoperatoria la linfocintigrafía y colorantes específicos, la observación permite realizar la disección linfática en la eventualidad que los linfonodos se hicieran palpables. Sin embargo, esta técnica no ha sido utilizada en el MMPE y, por lo tanto, pensamos que la técnica de Akiyama con la disección linfática de los territorios cervical, mediastínico y celíaco es la que ofrece las mejores perspectivas de curación. Otros tratamientos como radioterapia, quimioterapia e inmunoterapia obtienen beneficios limitados, y solo estarían recomendados cuando la resección quirúrgica no fuera posible $e^{4,11}$.

\section{Conclusión}

El MMPE es un tumor raro y muy agresivo. $\mathrm{Su}$ diagnóstico precoz, basado principalmente en el estudio histopatológico con inmunohistoquímica, permite detectarlo en una etapa localizada, susceptible al tratamiento resectivo. Si bien no existe evidencia suficiente que defina el manejo, la esofagectomía total ha sido asociada a mejores sobrevidas.

\section{Responsabilidades éticas}

Protección de personas y animales. Los autores declaran que para esta investigación no se han realizado experimentos en seres humanos ni en animales.

Confidencialidad de los datos. Los autores declaran que han seguido los protocolos de su centro de trabajo sobre la publicación de datos de pacientes.

Derecho a la privacidad y consentimiento informado. Los autores han obtenido el consentimiento informado de los pacientes y/o sujetos referidos en el artículo. Este documento obra en poder del autor de correspondencia.

\section{Financiación}

El presente trabajo no posee fuente de financiación.

\section{Conflicto de intereses}

Los autores declaran no tener ningún conflicto de intereses. 


\section{Bibliografía}

1. Caldwell CB, Bains MS, Burt M. Unusual malignant neoplasm of the esophagus. J Thorac Cardiovasc Surg. 1991;101:100-7.

2. Sabanathan S, Eng J, Pradhan GN. Primary malignant melanoma of the esophagus. Am J Gastroenterol. 1989;84:1475-9.

3. Weiner JP, Shao M, Wong A, Schwartz D, Schreiber D. Patterns of care and survival outcomes in the treatment of esophageal melanoma. Cancer Network ARS. 2016 [consultado 20 Nov 2016]. Disponible en: www.cancernetwork.com

4. Álvarez R, Funke R, Solís F, Molina H, Pacheco F, Farías H. Melanoma primario del esófago. Rev Chil Cir. 2009;61:16870
5. Butte J, Visscher A, de la Fuente H, Meneses M, Carrasco AM, Amaral H, et al. Melanoma primario del esófago tratado con esofagectomía. Casos clínicos. Rev Med Chile. 2010;138:77-81.

6. Yamazaki K, Ohmori T, Kumaga Y, Makuuchi H, Eyde B. Ultrastructure of esophageal melanocytosis. Virchows Arch A Pathol Anat Histopathol. 1991;418:51522.

7. Chalkidakis G, Wihlm JM, Morand G, Weill-Bousson M, Witz P. Primary malignant melanoma of the esophagus. Ann Thorac Surg. 1985;39:472-5.

8. Sunagawa $\mathrm{H}$, Nishimaki T, Shimoji H, Kuninaka K, Nakachi N, Kinjyou F. Primary malignant melanoma of the esophagus successfully treated by and esophagectomy followed by systemic chemotherapy. Esophagus. 2005;2:33-7.
9. Benito FG, Jiménez C, González R, Sáez MA, García A, Montero JM. Primary esophageal melanoma. Gastroenterol Hepatol. 1998;21:283-6.

10. Chang F, Deere H. Esophageal melanocytosis morphologic features and review of the literature. Arch Pathol Lab Med. 2006;130:552-7.

11. Archer HA, Owen WJ. Primary malignant melanoma of the esophagus. Dis Esophagus. 2000;13:320-3.

12. Joob AW, Haines GK, Kies MS, Shields TW. Primary malignant melanoma of the esophagus. Ann Thorac Surg. 1995;60:217-22.

13. Lin CY, Cheng YL, Huang WH, Lee SC. Primary malignant melanoma of the esophagus presenting with massive melena and hypovolemic shock. ANZ J Surg. 2002;72:62-4. 